OPEN ACCESS

Edited by:

Stephen Honeybul,

Sir Charles Gairdner Hospital,

Australia

Reviewed by:

J. Marc Simard

University of Maryland, Baltimore,

United States

Jacek Szczygielski,

Universitätsklinikum des Saarlandes,

Germany

Johannes Lemcke,

Unfallkrankenhaus Berlin, Germany

*Correspondence:

Gregory Hawryluk

gregory.hawryluk@hsc.utah.edu

Specialty section:

This article was submitted to

Neurotrauma

a section of the journal

Frontiers in Neurology

Received: 05 September 2018 Accepted: 07 January 2019

Published: 25 January 2019

Citation:

Shah A, Almenawer S and Hawryluk G (2019) Timing of Decompressive Craniectomy for Ischemic Stroke and Traumatic Brain Injury: A Review. Front. Neurol. 10:11. doi: 10.3389/fneur.2019.00011

\section{Timing of Decompressive Craniectomy for Ischemic Stroke and Traumatic Brain Injury: A Review}

\author{
Aatman Shah ${ }^{1}$, Saleh Almenawer ${ }^{2}$ and Gregory Hawryluk ${ }^{1 *}$ \\ ${ }^{1}$ Department of Neurosurgery, University of Utah School of Medicine, Salt Lake City, UT, United States, ${ }^{2}$ Division of \\ Neurosurgery, Hamilton Health Sciences and McMaster University, Hamilton, ON, Canada
}

While studies have demonstrated that decompressive craniectomy after stroke or $\mathrm{TBI}$ improves mortality, there is much controversy regarding when decompressive craniectomy is optimally performed. The goal of this paper is to synthesize the data regarding timing of craniectomy for malignant stroke and traumatic brain injury (TBI) based on studied time windows and clinical correlates of herniation. In stroke patients, evidence supports that early decompression performed within $24 \mathrm{~h}$ or before clinical signs of herniation may improve overall mortality and functional outcomes. In adult TBI patients, published results demonstrate that early decompressive craniectomy within $24 \mathrm{~h}$ of injury may reduce mortality and improve functional outcomes when compared to late decompressive craniectomy. In contrast to the stroke data, preliminary TBI data have demonstrated that decompressive craniectomy after radiographic signs of herniation may still lead to improved functional outcomes compared to medical management. In pediatric TBI patients, there is also evidence for better functional outcomes when treated with decompressive craniectomy, regardless of timing. More high quality data are needed, particularly that which incorporates a broader set of metrics into decision-making surrounding cranial decompression. In particular, advanced neuromonitoring and imaging technologies may be useful adjuncts in determining the optimal time for decompression in appropriate patients.

Keywords: TBI, stroke, decompressive hemicraniectomy, timing, herniation

\section{INTRODUCTION}

Decompressive craniectomy has been used to treat elevated intracranial pressure (ICP) resulting from various etiologies, especially ischemic and traumatic brain injuries. Given the inflexible confines of the skull, brain swelling from stroke or TBI can result in a compartment syndrome, increasing intracranial pressure (ICP). This can reduce cerebral perfusion pressure (CPP), cerebral blood flow (CBF), and oxygenation (1). If not acted upon, this can lead to brain ischemia, infarction, herniation, and death. There are various management strategies to treat elevated ICPs which include sedation, hyperventilation, hyperosmolar therapy, paralysis, hypothermia, barbiturates, and cerebrospinal fluid drainage (2). Decompressive craniectomy is a treatment option generally reserved for ICP elevation refractory to less invasive treatments (3).

Although decompressive craniectomy has been shown to effectively reduce ICP (4), there remains much controversy regarding its effect on overall clinical outcome, especially following TBI (5). Additionally, it is becoming clear that factors such as timing of decompressive craniectomy may 
play a significant role in determining the therapeutic benefit of this procedure. There are various studies providing insight into the optimal timing of decompressive craniectomy for victims of TBI and ischemic stroke and it is important for neurosurgeons to be aware of this data. The goal of this paper is to synthesize published findings regarding optimal timing for craniectomy for both malignant stroke and TBI in relation to time from injury and in relation to cerebral herniation.

\section{TIMING OF CRANIECTOMY AFTER ISCHEMIC STROKE}

\section{Decompressive Craniectomy for Stroke in the Animal Model}

Some animal data have suggested that early decompressive craniectomy could yield better functional outcomes than late decompression or non-operative management. In two animal studies with standardized experimental conditions, rats undergoing decompressive craniectomy after MCA infarction had a significantly better outcome and had a reduction in infarct size when compared to the non-surgical groups $(6,7)$. These studies were based on the hypothesis that avoiding herniation and mesencephalic ischemia would improve prognosis. Doerfler et al. concluded that the decompressive craniectomy groups demonstrated better mortality and neurologic outcome when compared to the non-surgical group (6). It was further concluded that the animals treated with very early decompression (within $4 \mathrm{~h}$ ) demonstrated significantly better neurologic outcomes and smaller infarct size compared to animals treated with later decompression. Forsting et al. also found that decompressive craniectomy improved outcomes, mortality, and infarct size when compared to the non-surgical group regardless of when the decompression occurred (7).

\section{Decompressive Craniectomy Within $48 \mathrm{~h}$ of Ischemic Stroke}

Decompressive craniectomy following acute ischemic stroke has been studied in three relatively recent human randomized controlled trials. These studies analyzed the effects of decompressive surgery on mortality and functional outcome after malignant hemispheric stroke. The subsequent pooling and meta-analysis of these studies has generated very important insights as will be described.

DECIMAL (Decompressive Craniectomy in Malignant Middle Cerebral Artery Infarcts) was published in 2007. It assigned 38 patients to undergo surgery or medical management within $30 \mathrm{~h}$ of their initial stroke (8) (see Table 1). When compared to the medical therapy cohort, the cohort that underwent decompressive craniectomy demonstrated a mortality rate that was more than halved, and a $50 \%$ increase in the proportion of patients with only moderate disability.

DESTINY (Decompressive Surgery for the Treatment of Malignant Infarction of the Middle Cerebral Artery) was also published in 2007. It enrolled 32 patients within $36 \mathrm{~h}$ of stroke (9). This randomized study demonstrated that craniectomy reduces mortality in large hemispheric stroke. Like DECIMAL, this study demonstrated a reduction in death rates in the surgical cohort, but also like DECIMAL the sample size of the DESTINY trial was not sufficient to draw conclusions regarding functional outcome.

Because the above studies were underpowered to assess differences in functional outcomes, the HAMLET (Hemicraniectomy After MCA Infarction With Life Threatening Edema Trial) trial was initiated. This third RCT was published in 2009 and was crucial in adding to the body of literature regarding decompressive surgery following acute ischemic stroke (10). HAMLET enrolled 64 patients up to $96 \mathrm{~h}$ after stroke. This RCT demonstrated that when decompressive craniectomy is delayed up to $96 \mathrm{~h}$, there was no improvement in functional outcomes in survivors. The percentage of patients with a mRS score less than or equal to three at 1 year follow up were comparable between the decompressive craniectomy and control group (25\% in both groups). It should be noted that three patients in the surgical group and three patients in the medical group had a fixed and dilated pupil on enrollment which means that roughly $20 \%$ of the study population demonstrated signs of herniation prior to treatment. Because $20 \%$ of this study population had already demonstrated signs of herniation, it can be argued that delayed craniectomy may be too late to impart any functional benefit.

A meta-analysis of the three studies was performed by Vahedi et al. on the patients treated with surgery within $48 \mathrm{~h}$ in the DECIMAL and DESTINY trials as well as the first 23 patients of the HAMLET trial (11). In this meta-analysis of 93 patients crossover was minimal: there was only one crossover from nonoperative treatment to decompressive surgery included in this analysis from the DESTINY trial. The results demonstrated increased favorable functional outcome compared to the medical cohort (11). In this paper, $43 \%$ of the decompressive craniectomy group had a modified Rankin scale (mRS) score of $0-3$ compared to $21 \%$ in the control group. It should be noted that from the human studies presented thus far, there have been no direct comparisons between outcomes of early vs. late decompressive craniectomy.

The findings from the meta-analysis performed by Vahedi et al. was further corroborated by the findings of Vibbert et al. This study contained 64 patients with acute ischemic stroke in the MCA territory who presented within $96 \mathrm{~h}$ of symptom onset. The patients were randomized to receive medical management or surgical intervention (3). The primary outcome was the modified Rankin scale (mRS) at 12 months which was stratified as good outcome (0-3) and severe disability or death (4-6). Twentyfour out of 32 patients in each arm had a mRS score $>3$ at 12 months, and rates of severe disability were also similar between groups. The risk of death was significantly reduced in the surgical group (absolute risk reduction of $38 \% ; P=0.002$ ). The authors performed subgroup analyses of patients who underwent surgery in $<48 \mathrm{~h}$ and patients who underwent surgery after $48 \mathrm{~h}$. For patients who underwent surgery within $48 \mathrm{~h}$ of stroke, the risk of death and an mRS score $>4$ were reduced (respectively: ARR, 59\%; 95\% CI, 33-84; ARR 30\%; 95\% CI, 1-59) (3).

Vibbert et al. then performed an updated meta-analysis with their cohort of patients and patients from the aforementioned DECIMAL, DESTINY, and HAMLET trials who underwent decompressive surgery within $48 \mathrm{~h}$ (3). Corroborating their prior 


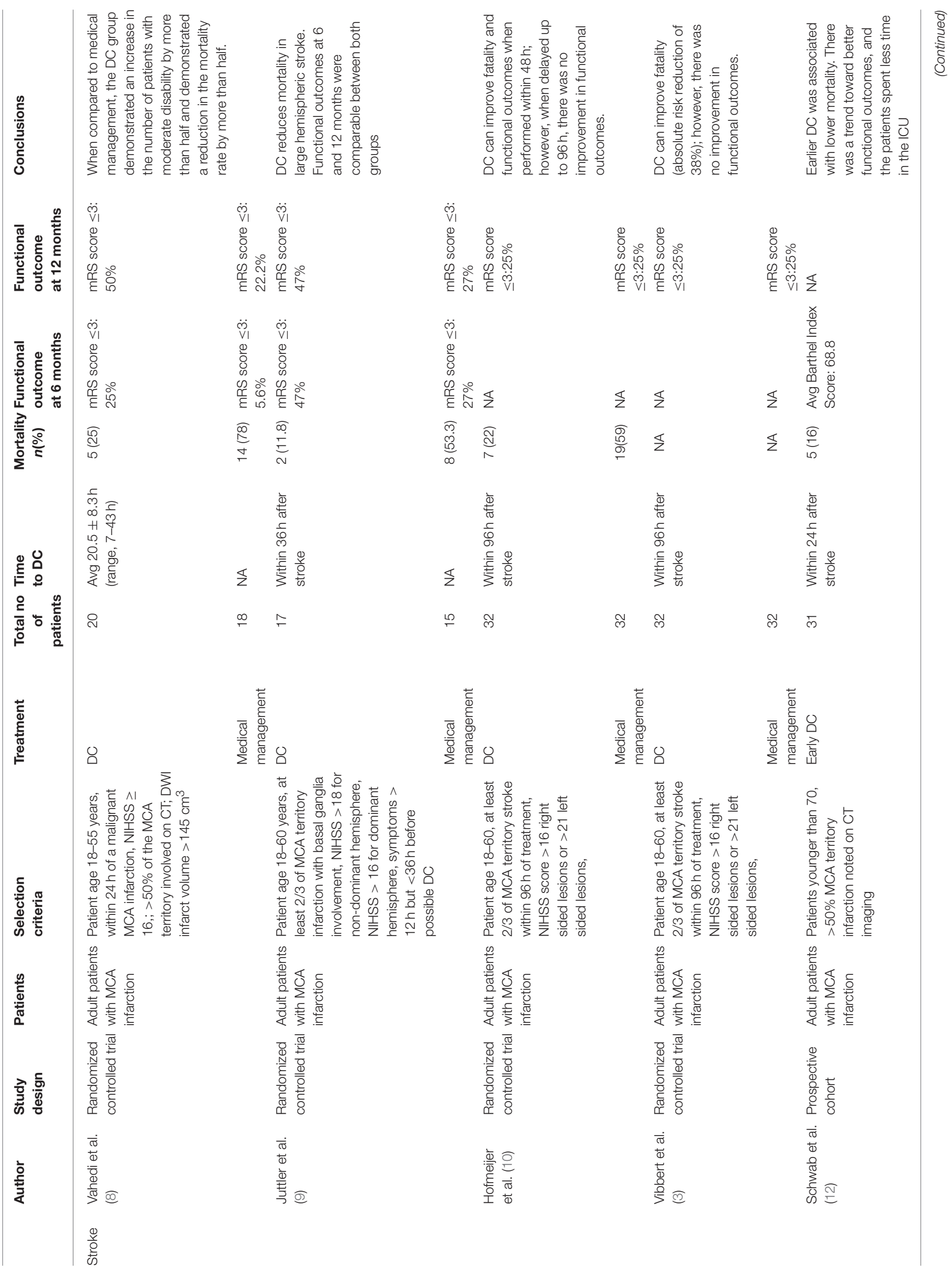




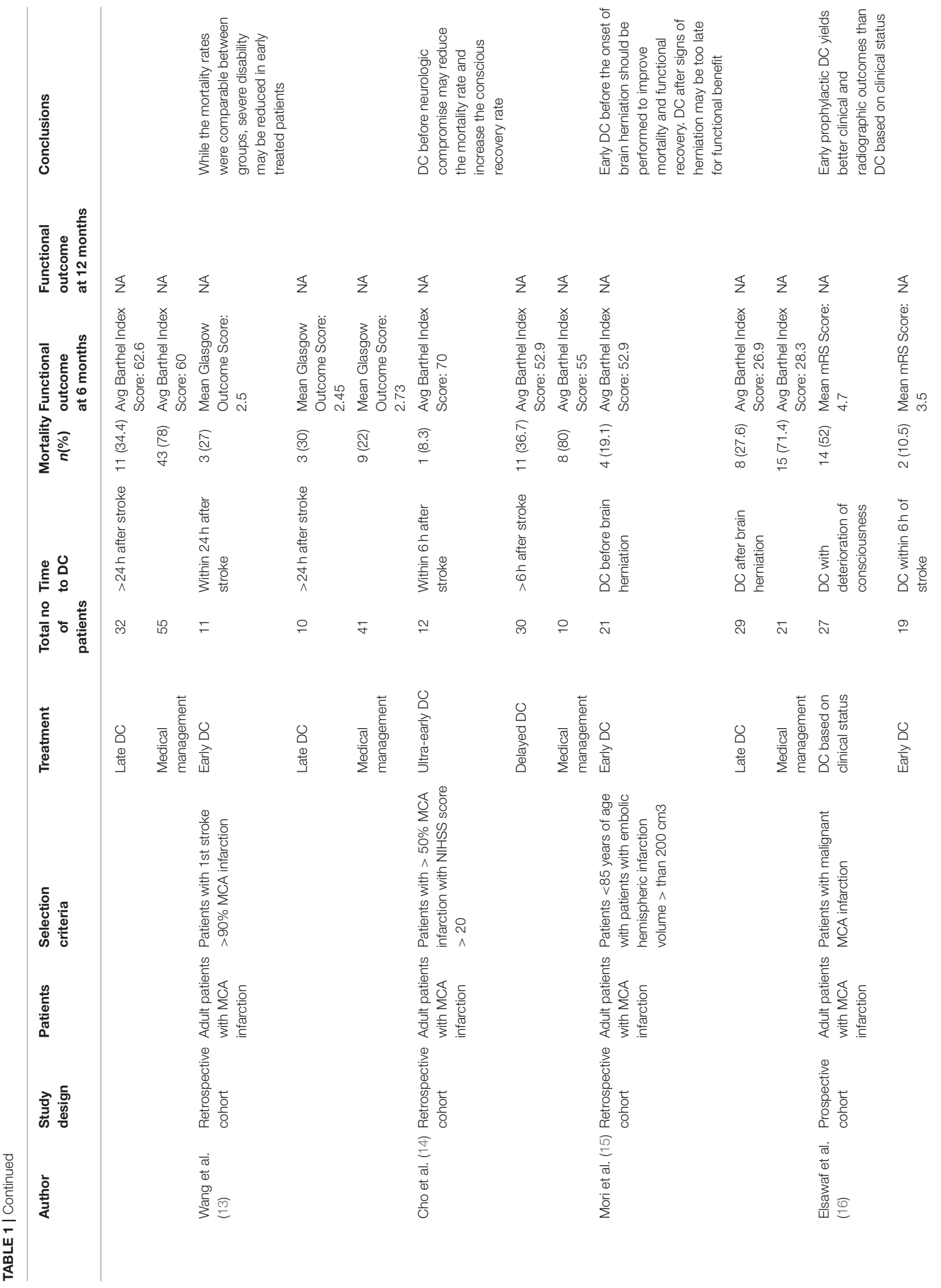


findings, the data suggest a significant reduction in risk of death (ARR, 49.9\%; 95\% CI, 33.9-65.9) and in the risk of severe disability at 12-months (ARR, 41.9\%; 95\% CI, 25.2-58.6). While not statistically significant, there was a notable trend toward reduction in risk of poor outcome (12 month mRS score >3-ARR, 16.3\%; 95\% CI, -0.1-33.1).

In summary, decompressive craniectomy within $96 \mathrm{~h}$ of malignant MCA stroke did not reduce poor outcomes at 1 year; however, there seems to be a trend toward reduction in death and moderate-to-severe disability (mRS score $>4$ ) when surgery was performed within $48 \mathrm{~h}$ from the stroke. It is possible that a significant portion of patients $96 \mathrm{~h}$ from an acute ischemic stroke may already exhibit herniation, and decompressive craniectomy at this time may be too late to impart functional benefit. Unfortunately, analysis of outcome in relation to herniation events was insufficiently described in these studies, and it is not possible to determine whether early surgery is beneficial due to avoiding herniation or whether there is a benefit to early surgery independent of herniation. The benefit of early surgery in the cohort by Vibbert et al. was not as great in magnitude as in HAMLET; however, this may have been due to a longer interval between symptom onset and surgical treatment in HAMLET (mean $31 \mathrm{~h}$ ) than in DECIMAL (mean $16 \mathrm{~h}$ ) and DESTINY (mean $24 \mathrm{~h}$ ).

\section{Decompressive Craniectomy Within $24 \mathrm{~h}$ of Ischemic Stroke}

Other data have suggested that early decompressive craniectomy within $24 \mathrm{~h}$ of stroke could yield even better functional outcomes. Schwab et al. conducted a prospective observational trial where the patient population was stratified by early craniectomy ( $<24 \mathrm{~h}$ after symptom onset) and late craniectomy ( $>24 \mathrm{~h}$ ), with additional comparison to a natural history group (12). Patients were included in the study if they had $>50 \%$ MCA territory infarction noted on CT imaging. The mean time between symptoms and surgery was $21 \mathrm{~h}$ (range, 8-42 h) in the early craniectomy group and $39 \mathrm{~h}$ (range, 6-112 h) in the late group. This difference approached statistical significance $(p=0.07)$. Mortality was $16 \%(5 / 31)$ in the early group, $34.4 \%(11 / 32)$ in the late group, and $78 \%(43 / 55)$ in the historical controls (12). The late group demonstrated uncal herniation in 24 of 32 patients $(75 \%)$ whereas only 4 of 31 patients $(13 \%)$ in the early group demonstrated uncal herniation. Length of stay in the ICU was 7.4 days for the early group and 13.3 days in the late treated group $(p=0.05)$. Functional outcome measured by the Barthel Index (BI) demonstrated a higher mean score for the early group with an average score of 70 vs. 62.6 in the late group. There was a trend toward better outcomes with early craniectomy, however, the data were not statistically significant. Overall, this study demonstrated that early craniectomy was an efficacious approach for treating malignant MCA infarction when the patients were treated before signs of herniation. The mortality rate was lower, there was a trend to better functional outcome, and the patients spent less time in the ICU.
The data presented by Schwab et al. were further corroborated by smaller series published by Wang et al. and Cho et al. In a retrospective study of 21 patients, Wang et al. compared the outcomes of early decompression $(<24 \mathrm{~h})$ to late decompression ( $>24 \mathrm{~h}$ ) (13). While the mortality rate was comparable, Wang et al. demonstrated that severe disability may be reduced in early treated patients. Cho et al. further corroborated this data, and demonstrated the positive results in association with ultra-early decompression defined as decompression within $6 \mathrm{~h}$ of symptom presentation (14). The Cho et al. study reported only a cohort of 52 patients and demonstrated that the acute mortality rate was statistically lower for the ultra-early group (8.3\%) compared to the delayed decompression group $(>6 \mathrm{~h}$ ) and the no surgery group (36.7 and $80 \%$, respectively, all $p$-values $<0.001)$. The ultra-early group also had better prognosis for conscious recovery (91.7\%) compared to the delayed decompression group and the no surgery group (55 and $0 \%$, respectively). While more data are needed, the published data give credence to the idea that early craniectomy performed within $24 \mathrm{~h}$ yields better mortality and functional outcomes. Moreover, this study suggests that the benefit to early surgery may not merely stem from an avoidance of herniation.

\section{Decompressive Craniectomy for Ischemic Stroke Based on Clinical Correlates of Herniation}

While the previous studies demonstrated benefit from early decompression, a key limitation was insufficient delineation of the role of herniation events in distinction to merely performing early surgery. Indeed, there have been more recent studies that indicate that the timing of craniectomy should be based on clinical features rather than on a strict temporal scale given the variations in when herniation events occur in the clinical course of different patients. A retrospective study by Mori et al. analyzed the outcomes of 71 patients with embolic hemispheric infarctions (infarct volume $>200 \mathrm{~cm}^{3}$ ) who were stratified into 3 groups: non-operative management, decompressive craniectomy after brain herniation (late surgery group), and decompressive craniectomy before brain herniation (early surgery group) (15). This study utilized the Glasgow Coma Scale (GCS), changes in mental status, and anisocoria as clinical indicators for herniation. The mortality at 1 and 6 months in the non-operative group was 61.9 and $71.4 \%$, respectively. The mortality at 1 and 6 months in the late surgery group was 17.2 and $27.6 \%$, respectively, $(p=0.01)$ and was even better in the early surgery group -4.8 and $19.1 \%$, respectively. The Glasgow Outcome Scale (GOS) and Barthel Index (BI) were employed as functional outcome measures at 6 months. The GOS scores of the early surgery group were better than those of the late surgery group $(p=0.05)$. The average BI score of the early surgery group $(52.9 \pm 34.2)$ were improved from those of the late surgery group $(26.9 \pm 30.4)(p=0.05)$. The late surgery group had a comparable BI score to the nonoperative group $(28.3 \pm 42.2)$, which indicates that surgery after signs of herniation may be too late to yield functional benefit. Mori et al. thus concluded that an effort should be made to perform early decompressive craniectomy before the onset of 
brain herniation in patients with malignant cerebral infarction. Mori et al. also concluded that embolic stroke patients with $>200$ $\mathrm{cm}^{3}$ volume of infarction and shift of the midline structures on a follow-up CT 2 days after ictus are more likely to herniate and would benefit from decompressive craniectomy.

Mori et al. advanced the field by conceptualizing outcome in relation to clinical indicators of herniation. With this in mind, Elsawaf et al. published a recent and important prospective study comparing outcomes of early decompression (within $6 \mathrm{~h}$ of presentation) and decompression based on clinical features of deterioration. Forty-six patients with large hemispheric MCA infarction were divided randomly into two groups: Group I in which patients were followed until deterioration of level of consciousness, and Group II in which patients were operated within $6 \mathrm{~h}$ of presentation regardless of clinical signs of deterioration or radiographic features (16). While both groups demonstrated improvement in conscious level, motor power, and functional outcome, there was significant improvement $(p<0.05)$ in functional outcome in group II based on the mRS. Group I demonstrated increased progression of infarct volume when compared to Group II, and also had a morality of $52 \%$ due to delay in surgery compared to $10 \%$ in Group II. This study found better clinical and radiographic outcomes for patients with large hemispheric MCA infarction who were operated on prophylactically within $6 \mathrm{~h}$ of infarction without waiting for deterioration of level of consciousness.

While there are concerns that very early decompression surgery might potentially be unnecessary, the presented data demonstrate that decompression after the onset of herniation symptoms is less effective, or may even be ineffective in reducing mortality and improving neurological outcome. While more data are required, current studies suggest that stroke patients with malignant infarction $>200 \mathrm{~cm}^{3}$ and follow up CT at 2 days from symptom onset which demonstrate shift of the midline structures are likely to herniate and would benefit from early decompressive craniectomy.

\section{TIMING OF CRANIECTOMY AFTER TRAUMATIC BRAIN INJURY}

\section{Decompressive Craniectomy for Traumatic Brain Injury (TBI) in the Animal Model}

Preliminary data from TBI animal models treated with decompressive craniectomy have suggested that decompressive craniectomy could reduce edema formation and prevent secondary expansion of the original contusion when compared to non-operative management. Zweckberger et al. utilized a controlled cortical impact model of TBI in a cohort of mice to study the influence of decompressive craniectomy on secondary contusion expansion and brain edema formation, and to determine optimal timing of decompressive craniectomy (25). It was determined that in the surgical groups, there was no secondary expansion of the original contusion and there was a $52 \%$ reduction of brain edema compared to the non-surgical group. These benefits were seen with decompressive craniectomy when performed up to $3 \mathrm{~h}$ after the initial trauma. Tomura et al utilized a fluid percussion injury model of TBI in a cohort of rats to investigate the influence of decompressive craniectomy on post traumatic brain edema formation. It was found that the non-surgical group demonstrated less cortical water content and greater AQP4 expression when compared to the decompressive craniectomy group.

\section{Decompressive Craniectomy More Than 24h After TBI}

Although there is some controversy regarding the use of decompressive craniectomy in ischemic stroke patients, the use of decompressive craniectomy following human TBI has certainly been more controversial. Three RCTs have analyzed the outcomes of TBI patients after late decompressive craniectomy (more than $24 \mathrm{~h}$ from the injury) (see Table 2). The DECRA (Decompressive Craniectomy in Diffuse Traumatic Brain Injury) trial published by Cooper et al. in 2011 was a landmark RCT which informed the outcomes of TBI patients with diffuse injuries who were treated with decompressive craniectomy within $72 \mathrm{~h}$ of injury (17). In this study, 155 patients with refractory ICPs $>20 \mathrm{mmHg}$ for $15 \mathrm{~min}$ within a 1 -h period were randomized into a decompressive craniectomy group (bifrontal decompressive craniectomy) or a maximal medical management group. On average, the time from injury to surgery was $38.1 \mathrm{~h}$, with a range of 27.1-55.0 h. In this study, Cooper et al. determined that bifrontal decompressive craniectomy decreases ICP and the length of stay in the intensive care unit, but is associated with more unfavorable outcomes. There are, however, some criticisms involving the DECRA trial. First, the randomization was uneven between the 2 groups. There were more patients with non-reactive pupils in the decompressive craniectomy group than the medical therapy group (27 vs. $12 \%$, respectively $[p=0.04])$. It can be argued that more patients in the decompressive craniectomy group already demonstrated signs of herniation prior to treatment which may obfuscate the therapeutic benefit from a decompressive craniectomy. Indeed, the harm associated with decompression was no longer statistically significant when a statistical control for unreactive pupils was performed. Other issues included the relatively small sample size and that only bifrontal decompressive craniectomy without falx sectioning was allowed. Some researchers believe DECRA was too aggressive and that ICP elevations should have been sustained for longer durations prior to considering surgery. Lastly, there were no standardized rehabilitation protocol for the enrolled patients.

After the DECRA trial, the therapeutic effect of decompressive craniectomy in TBI patients remained unclear, particularly in patients with focal pathology and when a lateral decompression is performed. In 2016, Hutchinson el al. published a multicenter (48 centers, 19 countries) RCT study named RESCUEicp (Trial of Decompressive Craniectomy for Traumatic Intracranial Hypertension) in which a cohort of 408 patients with TBI and refractory elevated ICP (>25 $\mathrm{mmHg}$ for at least $1 \mathrm{~h}$ ) were randomized into a decompressive craniectomy group or a maximal medical therapy group (18). In this pragmatic study, $44 \%$ of patients were enrolled after $72 \mathrm{~h}$. RESCUEicp was 


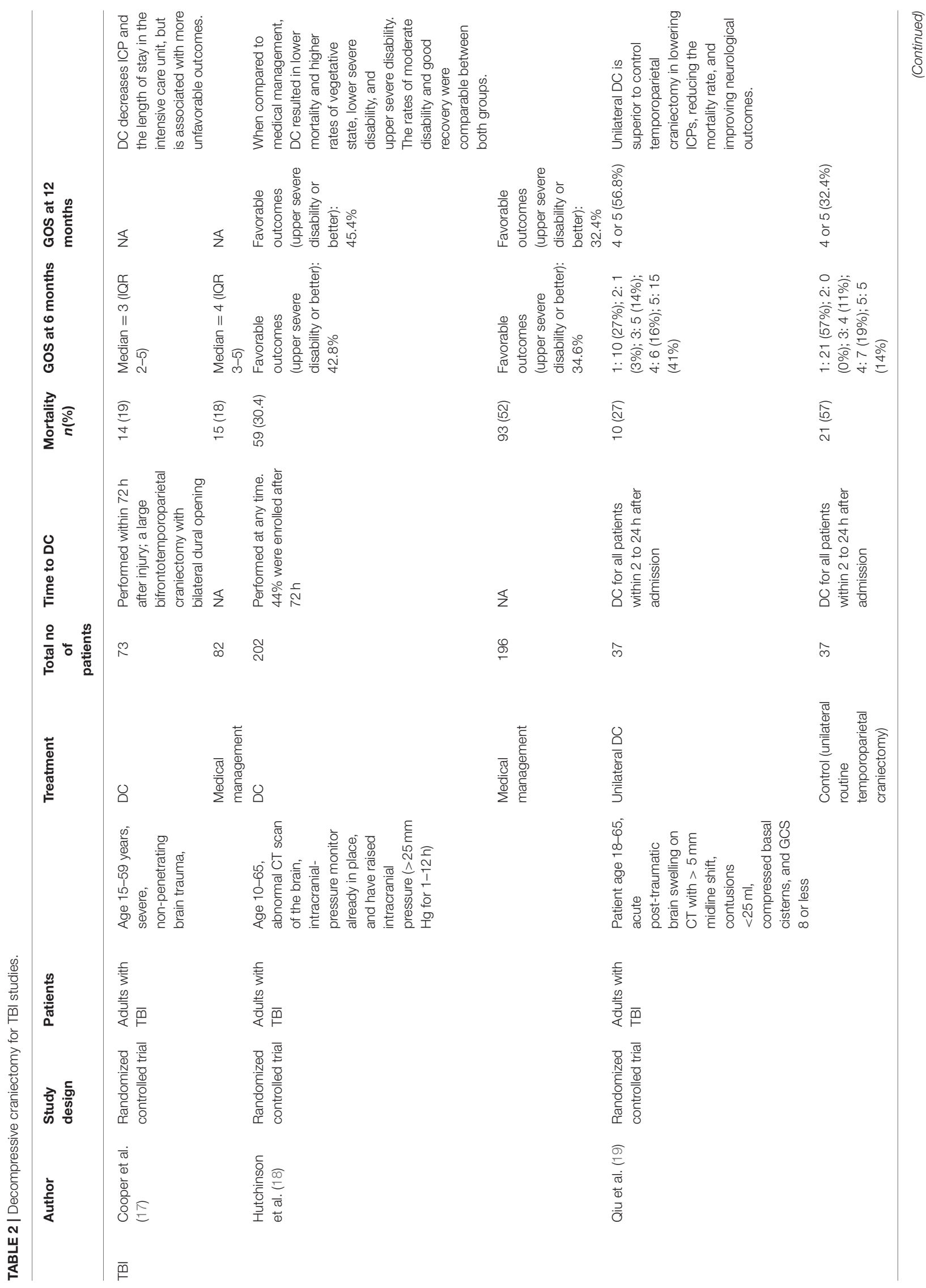




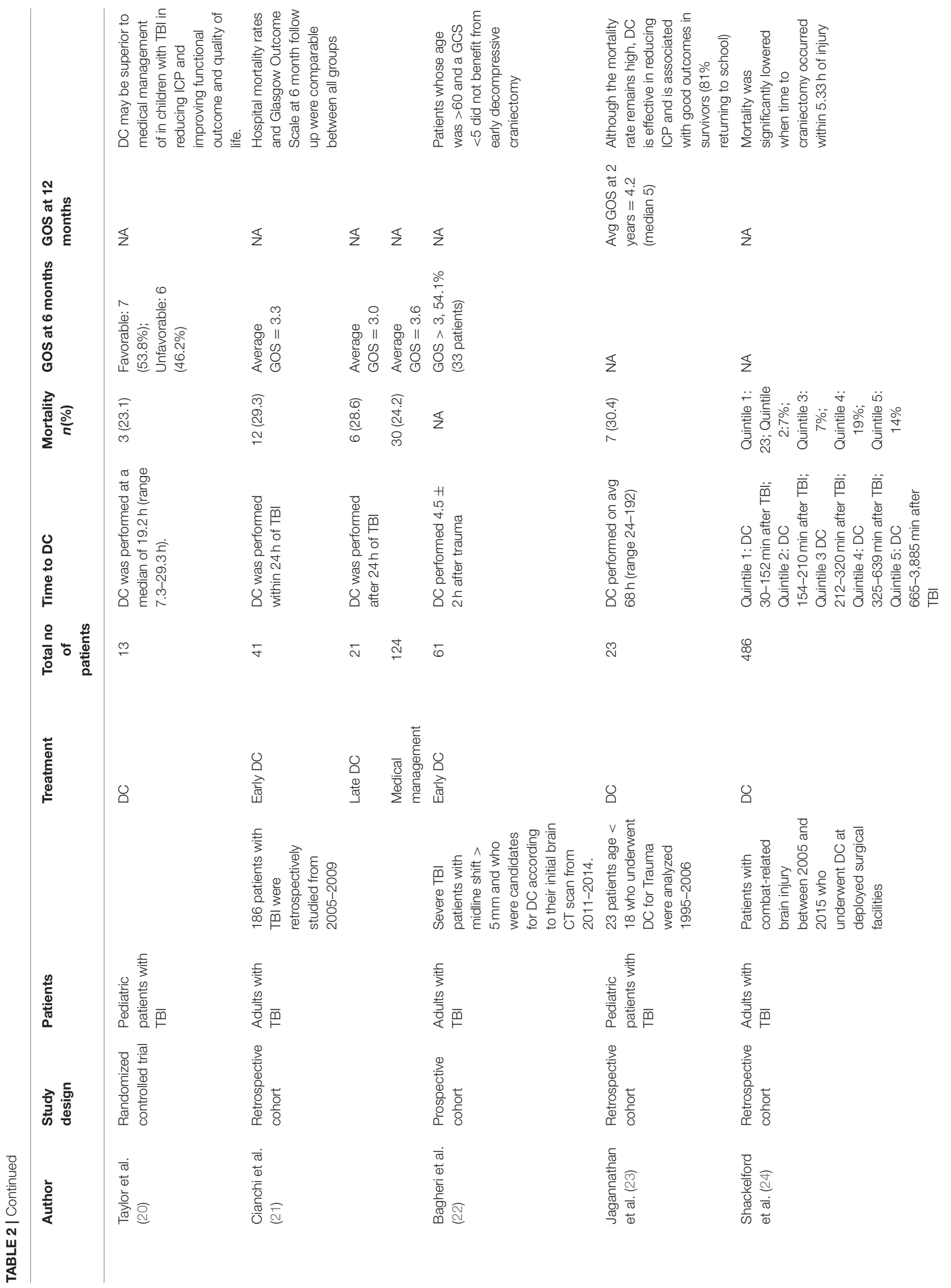


intended to study a distinct population of patients as compared with DECRA. The DECRA trial looked at decompression within $72 \mathrm{~h}$ after diffuse TBI, whereas the RESCUEicp trial analyzed decompressive craniectomy as salvage therapy for refractory intracranial hypertension. Moreover, patients with intracranial hematoma were not included in DECRA trial, but accounted for about $20 \%$ of the RESCUEicp trial. Unilateral craniectomy was not permitted in DECRA trial but was allowed in the RESCUEicp trial. At 6 months, the patients in RESCUEicp's decompressive craniectomy group exhibited lower mortality but higher rates of vegetative state, "lower severe" disability, "upper severe" disability, and comparable rates of moderate disability and "good recovery" when compared to the medical management group. It should also be noted that in the subgroup analysis comparing decompressive craniectomy performed before $72 \mathrm{~h}$ and at $72 \mathrm{~h}$ or more, there were no differences noted in functional outcomes. In interpreting this trial it is important to consider that $37.2 \%$ (73 patients) of the patients in the medical group ultimately underwent decompressive craniectomy. Notably, ten patients were excluded from analysis due withdrawal/lack of valid consent. Seven additional patients in the medical group were lost to follow-up. It is particularly important to consider that the majority of the patients in the RESCUEicp trial had diffuse injuries $(78.6 \%$ of all study patients between the surgical and medical therapy groups) and underwent bifrontal decompressions (81.3\% of the surgical group) despite the intent to enroll a distinct population from DECRA. With this in mind, the authors of this manuscript view RESCUEicp as confirming the findings of DECRA without substantially informing the use of decompressive craniectomy in patients with focal pathology, and the role of lateral decompressions.

Due to the paucity of data analyzing the importance of timing of decompressive craniectomy in outcomes of TBI patients, a meta-analysis published by Zhang et al. demonstrated that early decompressive craniectomy within $36 \mathrm{~h}$ could result in better prognosis based on the Glasgow Outcome Scale scores at 6 months when compared to patients operated on $>36 \mathrm{~h}$ from the initial injury (5). The meta-analysis included 10 studies with four randomized controlled trials. On sub-group analysis, Zhang et al. determined that decompressive craniectomy could reduce mortality rate, lower ICPs, decrease ICU stay, but could also increase complication rate.

\section{Decompressive Craniectomy Within $24 \mathrm{~h}$ of TBI}

While the aforementioned studies analyzed outcomes following decompressive craniectomy performed more than $24 \mathrm{~h}$ from time of injury, there have been efforts to analyze outcomes in TBI patients treated with early decompressive craniectomy within $24 \mathrm{~h}$ of injury. To that end, Cianchi et al. published their findings from a retrospective analysis which looked at the outcomes of early vs. late decompressive craniectomy compared to maximal medical management in treating TBI patients (21). In this study, 186 TBI patients were divided into early decompressive craniectomy (surgery within $24 \mathrm{~h}$ of TBI), late decompressive craniectomy (surgery after $24 \mathrm{~h}$, on average 7.7 days after TBI), and maximal medical management groups. Hospital mortality rates and Glasgow Outcome Scale at 6 month follow up were comparable between all groups; however, the 6 month mortality rate was significantly less for the maximal medical management group compared to the early and late decompressive craniectomy groups $(29,48.8,42.9 \%$, respectively $[p=0.02])(21)$. One of the main limitations of this analysis is the retrospective study design. Inherently, patients in the control group had intracranial pressures that were adequately treated with medical therapy whereas patients who received decompressive craniectomy failed medical therapy. It is therefore reasonable to conclude that the patients who underwent decompressive craniectomy had, on average, a more severe TBI. A more appropriate control group would include patients who were non-responders to medical treatment who were not treated with late decompressive craniectomy; however, there are obvious ethical considerations limiting such a study design.

To better address the importance of early decompressive craniectomy in TBI patients, Qiu et al. published an RCT analyzing the outcomes of early decompressive craniectomy in TBI patients (19). Seventy-four patients were randomized to either unilateral decompressive craniectomy or a control group which consisted of a unilateral routine temporoparietal craniectomy. All surgery occurred between 2 and $24 \mathrm{~h}$ (average $5.8 \mathrm{~h}$ ) after admission. Enrolled patients needed to demonstrate $>5 \mathrm{~mm}$ of midline shift on CT and compression of the basal cisterns. In this RCT, the entire cohort had progressed to some form of radiographic herniation. The mortality rates at 1 month after treatment were $27 \%$ in the unilateral decompressive craniectomy group and $57 \%$ in the control group. At 1 year follow up, good neurological outcome (GOS Score of 4-5) were $56.8 \%$ for the decompressive craniectomy group and $32.4 \%$ for the control group $(p=0.035)$. In contrast to the previous stroke studies which demonstrated that decompressive craniectomy after herniation does not confer any functional benefit, Qiu et al. concluded that unilateral decompressive craniectomy after radiographic signs of herniation may be superior to the control group at lowering ICPs, reducing the mortality rate, and improving functional outcome. It should be reiterated that all surgeries were performed within $24 \mathrm{~h}$ which is considered to be "early" compared to the timing of decompression reported in most of the TBI in the literature.

Bagheri et al. corroborated the findings of Qiu et al. and published their findings from a prospective study of 61 patients who underwent rapid decompressive craniectomy (within $4.5 \pm$ $2 \mathrm{~h}$ ) after trauma to assess factors associated with prognosis and outcome (22). Of the 61 patients, $54.1 \%$ demonstrated favorable functional outcomes; however, patients with ages older than 60 years, bilateral non-reactive mydriasis, critical head injury $(\mathrm{GCS}<5)$, or with $>1 \mathrm{~cm}$ midline shift had worse outcomes. Bagheri argued that patients whose age was $>60$ and a GCS $<5$ did not benefit from early decompressive craniectomy.

Lastly, a large retrospective review involving 486 patients with combat related TBI who underwent decompressive craniectomy demonstrated that decompression within $5.33 \mathrm{~h}$ from TBI was associated with improved survival (24). The mortality of the patients were reported by time interval related quintiles: quintile 
1 was defined as decompressive craniectomy 30-152 min after TBI, quintile 2 was defined as decompressive craniectomy 154$210 \mathrm{~min}$ after TBI, quintile 3 was defined as decompressive craniectomy $212-320 \mathrm{~min}$ after TBI, quintile 4 was defined as decompressive craniectomy 325-639 min after TBI, and quintile 5 was defined as decompressive craniectomy 665-3,885 min after TBI. The postoperative mortality was $23,7,7,19$, and $14 \%$ respectively. Mortality was significantly lowered when time to craniectomy occurred within $5.33 \mathrm{~h}$ of injury. While providing some insight into the possible importance of ultraearly decompressive craniectomy on survival, the retrospective design and the lack of long term functional outcome data limits the conclusions that can be drawn from this study.

Although more research is needed, decompressive craniectomy remains a frequently performed treatmentgenerally of last resort-for many patients with severe TBI. Much additional research is needed to optimize how and when this surgery is performed. In contrast to the findings in the stroke data, preliminary data for TBI studies demonstrate that decompressive craniectomy after acute herniation may still be beneficial in improving mortality and functional outcomes. Although more data are needed, TBI patients treated with early decompressive craniectomy seem to have lower mortality and potentially better functional outcomes than TBI patients treated with late decompressive craniectomy. As with the stroke data, the analysis of outcome for TBI patients in relation to herniation events was insufficiently described in relevant studies, and it is not possible to determine whether early surgery is beneficial due to avoiding herniation or whether there is a benefit to early surgery independent of herniation. While the larger RCTs indicate that decompressive craniectomy may increase the survival rate and concomitantly increase rates of severe disability including vegetative state, subsequent trials with a shorter duration to decompressive craniectomy have demonstrated improved functional outcomes and less mortality.

\section{Early Decompressive Craniectomy in Pediatric TBI Patients}

Some published data demonstrate that early decompressive craniectomy may be beneficial in the pediatric population. To that end, Taylor et al. published the only RCT analyzing outcomes of early decompressive craniectomy in the pediatric population (20). Twenty-seven children who had sustained ICP elevation after TBI were randomized to the medical management group or the decompressive craniectomy group. Early bitemporal decompressive craniectomy was performed for the surgical group at a median of $19.2 \mathrm{~h}$ (range $7.3-29.3 \mathrm{~h}$ ) from the time of TBI. Outcome was assessed 6 months after the TBI using a modification of the Glasgow Outcome Score (GOS) and the Health State Utility Index. At 6 months, $54 \%$ of children in the decompressive craniectomy group had good outcomes or mild disability at 6 months compared to $14 \%$ of children in the control group. Taylor et al. concluded that in pediatric TBI patients with refractory ICPs, patients treated with early decompressive craniectomy are more likely to have reduced ICPs and improved functional outcome than children treated with maximal medical therapy alone. While this is the only RCT published regarding decompressive craniectomy in the pediatric population, this study has received some criticism because it involved an unusual decompressive surgery in which the dura was not opened, and because it accrued a small number of patients over a long study period.

Jagannathan et al. corroborated the findings from Taylor et al. in their retrospective review on the outcomes of 23 pediatric patients who underwent decompressive craniectomy for TBI (23). The time to decompressive craniectomy was on average $68 \mathrm{~h}$ (range 24-192). Despite having longer time to decompressive craniectomy compared to Taylor et al., the mean GOS score at the 2-year follow-up examination was 4.2 (median 5). At latest follow up, $81 \%$ of the patients returned to school, and only $18 \%$ were dependent on caregivers. It should be noted that the outcomes in the Taylor et al. cohort were analyzed at 6 months, whereas the outcomes in the manuscript by Jagannathan et al. were analyzed at 2 year follow up, substantially confounding a comparison of the two trials. Although more data are needed, it is possible that earlier decompression may not be as important in improving long term outcomes in the pediatric population as has been shown in the adult population.

With the limited data at hand, it appears that the pediatric population has better functional outcomes with decompressive craniectomy regardless of timing when compared to medical management. Unfortunately, direct comparisons between early and late decompressive craniectomy have not been made in the pediatric population. Larger RCTs with direct comparisons will be needed to determine if timing plays a role improving outcomes in the pediatric population.

\section{Future Directions: Decompressive Craniectomy Based on Biologic and Radiographic Metrics}

While there are data validating the benefits of early craniectomy based on specific time windows and clinical correlates of herniation, there are growing data that there may be other biologic and radiographic metrics to help guide timing of decompressive craniectomy for TBI and stroke. Strict control of intracranial pressures and cerebral perfusion pressures alone does not necessarily prevent cerebral hypoxia (26). Recent data have demonstrated that measurement of brain tissue oxygen tension $\left(\mathrm{P}_{\mathrm{bt}} \mathrm{O}_{2}\right)$ may more precisely measure the adequacy of cerebral perfusion, and could be a useful adjunct for deciding on the timing of decompressive craniectomy (27). A $\mathrm{P}_{\mathrm{bt}} \mathrm{O}_{2}$ below $20 \mathrm{mmHg}$ has been associated with poor outcomes in TBI patients (28). Reithmeier et al. published data on the effects of decompressive craniectomy on ICPs and $\mathrm{P}_{b t} \mathrm{O}_{2}$ based on the continuous monitoring of 15 patients and determined that $\mathrm{P}_{\mathrm{bt}} \mathrm{O}_{2}$ monitoring could serve as a useful tool for timing craniectomy (2). One criticism of $\mathrm{P}_{b t} \mathrm{O}_{2}$ is that its measurements are based on data from the confines of a small volume of brain tissue which may not adequately reflect the oxygenation of a larger expanse of compromised brain. Other potentially useful biologic metrics include the pressure reactivity index $(\mathrm{PRx})$ which is the correlation coefficient between mean intracranial pressure 
(ICP) and mean arterial blood pressure. This could be used as a surrogate marker of cerebrovascular impairment (29). There have also been preliminary data suggesting that surrogates for bloodbrain-barrier disruption, defined by a ratio of total CSF protein concentrations to total plasma protein concentration, may also be useful for prognosis and treatment (30). Advances in imaging modalities may also be utilized to guide the treatment trajectory. The infarct growth rate (IGR) between two CT scans may also be a useful tool for timing craniectomy. Kamran et al. published a retrospective, multicenter cross-sectional study of 182 patients to identify factors for selecting the timing of craniectomy (31). The IGR on the second CT was one of the five factors identified as having the strongest association with craniectomy. Patients who survived without surgery had the slowest IGRs. On another retrospective cohort of 137 patients, Kamran et al. demonstrated that IGR was identified as an independent predictor of early surgery (32). The second infarct growth rate [IGR2] $>7.5 \mathrm{ml} / \mathrm{hr}$ was associated with surgery under $48 \mathrm{~h}$. Both first infarct growth rate [IGR1] and second infarct growth rate [IGR2] were nearly double in patients with early surgery within $48 \mathrm{~h}$. Although more data are needed, monitoring the infarct growth rate could help determine when a neurosurgeon should pursue decompression. While promising, these biologic and radiographic metrics still require more data before they are used to counsel patients regarding treatment course and prognosis.

\section{CONCLUSION}

Although there is much controversy surrounding optimal timing of decompressive craniectomy in patients with stroke and TBI, data have suggested that early decompression within

\section{REFERENCES}

1. Kolias AG, Kirkpatrick PJ, Hutchinson PJ. Decompressive craniectomy: past, present and future. Nat Rev Neurol. (2013) 9:405-15. doi: 10.1038/nrneurol.2013.106

2. Reithmeier T, Lohr M, Pakos P, Ketter G, Ernestus RI. Relevance of ICP and ptiO2 for indication and timing of decompressive craniectomy in patients with malignant brain edema. Acta Neurochir. (2005) 147:947-51; discussion: 952. doi: 10.1007/s00701-005-0543-1

3. Vibbert M, Mayer SA. Early decompressive hemicraniectomy following malignant ischemic stroke: the crucial role of timing. Curr Neurol Neurosci Rep. (2010) 10:1-3. doi: 10.1007/s11910-009-0081-y

4. Bongiorni GT, Hockmuller MCJ, Klein C, Antunes ACM. Decompressive craniotomy for the treatment of malignant infarction of the middle cerebral artery: mortality and outcome. Arq Neuropsiquiatr. (2017) 75:424-8. doi: 10.1590/0004-282x20170053

5. Zhang D, Xue Q, Chen J, Dong Y, Hou L, Jiang Y, et al. Decompressive craniectomy in the management of intracranial hypertension after traumatic brain injury: a systematic review and meta-analysis. Sci Rep. (2017) 7:8800. doi: 10.1038/s41598-017-08959-y

6. Doerfler A, Forsting M, Reith W, Staff C, Heiland S, Schabitz WR, et al. Decompressive craniectomy in a rat model of "malignant" cerebral hemispheric stroke: experimental support for an aggressive therapeutic approach. J Neurosurg. (1996) 85:853-9. doi: 10.3171/jns.1996.85.5.0853

7. Forsting M, Reith W, Schabitz WR, Heiland S, von Kummer R, Hacke W, et al. Decompressive craniectomy for cerebral infarction. An experimental study in rats. Stroke (1995) 26:259-64. doi: 10.1161/01.STR.26.2.259
$24 \mathrm{~h}$ has a tendency to improve mortality and functional outcomes for both conditions when compared to decompression performed after $24 \mathrm{~h}$. In stroke patients, decompression before clinical signs of herniation yields improved functional outcomes when compared to decompression after clinical signs of herniation. Surgery after clinical deterioration may be too late to impart any functional benefit in stroke patients. In contrast to the stroke data, preliminary TBI data have demonstrated that decompressive craniectomy after signs of herniation may still lead to improved functional outcomes compared to medical management. In adult TBI patients, early decompressive craniectomy within $24 \mathrm{~h}$ may improve mortality and functional outcomes when compared to decompressive craniectomy performed $>24 \mathrm{~h}$. In fact, data from RCTs suggest that late decompressive craniectomy for TBI may result in worse functional outcomes than maximal medical therapy. In pediatric TBI patients, patients also had better functional outcomes when treated with decompressive craniectomy regardless of timing. High quality studies better informing the timing and indications for decompressive craniectomy are needed for both ischemic stroke and TBI. The additional data provided by imaging and advanced neuromonitoring could also be useful adjuncts in guiding decision making.

\section{AUTHOR CONTRIBUTIONS}

AS: Conceptualization of the manuscript, literature review, data analysis, and manuscript writing. SA: Data analysis and revision consultant. GH: Supervised, edited/wrote the manuscript and literature/data analysis.

8. Vahedi K, Vicaut E, Mateo J, Kurtz A, Orabi M, Guichard JP, et al. Sequentialdesign, multicenter, randomized, controlled trial of early decompressive craniectomy in malignant middle cerebral artery infarction (DECIMAL Trial). Stroke (2007) 38:2506-17. doi: 10.1161/STROKEAHA.107.485235

9. Juttler E, Schwab S, Schmiedek P, Unterberg A, Hennerici M, Woitzik J, et al. Decompressive surgery for the treatment of malignant infarction of the middle cerebral artery (DESTINY): a randomized, controlled trial. Stroke (2007) 38:2518-25. doi: 10.1161/STROKEAHA.107.485649

10. Hofmeijer J, Kappelle LJ, Algra A, Amelink GJ, van Gijn J, van der Worp $\mathrm{HB}$, et al. Surgical decompression for space-occupying cerebral infarction (the Hemicraniectomy After Middle Cerebral Artery infarction with Lifethreatening Edema Trial [HAMLET]): a multicentre, open, randomised trial. Lancet Neurol. (2009). 8:326-33. doi: 10.1016/S1474-4422(09)70047-X

11. Vahedi K, Hofmeijer J, Juettler E, Vicaut E, George B, Algra A, et al. Early decompressive surgery in malignant infarction of the middle cerebral artery: a pooled analysis of three randomised controlled trials. Lancet Neurol. (2007) 6:215-22. doi: 10.1016/S1474-4422(07)70036-4

12. Schwab S, Steiner T, Aschoff A, Schwarz S, Steiner HH, Jansen O, et al. Early hemicraniectomy in patients with complete middle cerebral artery infarction. Stroke (1998) 29:1888-93. doi: 10.1161/01.STR.29.9.1888

13. Wang KW, Chang WN, Ho JT, Chang HW, Lui CC, Cheng MH, et al. Factors predictive of fatality in massive middle cerebral artery territory infarction and clinical experience of decompressive hemicraniectomy. Eur J Neurol. (2006) 13:765-71. doi: 10.1111/j.1468-1331.2006.01365.x

14. Cho DY, Chen TC, Lee HC. Ultra-early decompressive craniectomy for malignant middle cerebral artery infarction. Surg Neurol. (2003) 60:227-32; discussion 232-223. doi: 10.1016/S0090-3019(03)00266-0 
15. Mori K, Nakao Y, Yamamoto T, Maeda M. Early external decompressive craniectomy with duroplasty improves functional recovery in patients with massive hemispheric embolic infarction: timing and indication of decompressive surgery for malignant cerebral infarction. Surg Neurol. (2004) 62:420-9; discussion 429-30. doi: 10.1016/S0090-3019(04)00151-X

16. Elsawaf A, Galhom A. Decompressive craniotomy for malignant middle cerebral artery infarction: optimal timing and literature review. World Neurosurg. (2018) 116:e71-8. doi: 10.1016/j.wneu.2018.04.005

17. Cooper DJ, Rosenfeld JV, Murray L, Arabi YM, Davies AR, D'Urso P, et al. Decompressive craniectomy in diffuse traumatic brain injury. $N$ Engl J Med. (2011) 364:1493-502. doi: 10.1056/NEJMoa1102077

18. Hutchinson PJ, Kolias AG, Timofeev IS, Corteen EA, Czosnyka M, Timothy J, et al. Trial of decompressive craniectomy for traumatic intracranial hypertension. $N$ Engl J Med. (2016) 375:1119-30. doi: 10.1056/NEJMoa1605215

19. Qiu W, Guo C, Shen H, Chen K, Wen L, Huang H, et al. Effects of unilateral decompressive craniectomy on patients with unilateral acute post-traumatic brain swelling after severe traumatic brain injury. Crit Care (2009) 13:R185. doi: $10.1186 /$ cc8178

20. Taylor A, Butt W, Rosenfeld J, Shann F, Ditchfield M, Lewis E, et al. A randomized trial of very early decompressive craniectomy in children with traumatic brain injury and sustained intracranial hypertension. Childs Nerv Syst. (2001) 17:154-62. doi: 10.1007/s003810000410

21. Cianchi G, Bonizzoli M, Zagli G, di Valvasone S, Biondi S, Ciapetti M, et al. Late decompressive craniectomyafter traumatic brain injury: neurological outcome at 6 months after ICU discharge. J Trauma Manag Outcomes (2012) 6:8. doi: 10.1186/1752-2897-6-8

22. Bagheri SR, Alimohammadi E, Saeidi H, Fatahian R, Sepehri P, Soleimani $\mathrm{P}$, et al. Decompressive craniectomy in traumatic brain injury: factors influencing prognosis and outcome. Iran J Neurosurg. (2017) 3:21-6. doi: 10.29252/irjns.3.1.21

23. Jagannathan J, Okonkwo DO, Dumont AS, Ahmed H, Bahari A, Prevedello DM, et al. Outcome following decompressive craniectomy in children with severe traumatic brain injury: a 10-year singlecenter experience with long-term follow up. J Neurosurg. (2007) 106:268-75. doi: $10.3171 /$ ped.2007.106.4.268

24. Shackelford SA, Del Junco DJ, Reade MC, Bell R, Becker T, Gurney J, et al. Association of time to craniectomy with survival in patients with severe combat-related brain injury. Neurosurg Focus (2018) 45:E2. doi: 10.3171/2018.9.FOCUS18404
25. Zweckberger K, Eros C, Zimmermann R, Kim SW, Engel D, Plesnila N. Effect of early and delayed decompressive craniectomy on secondary brain damage after controlled cortical impact in mice. J Neurotrauma (2006) 23:1083-93. doi: 10.1089/neu.2006.23.1083

26. White H, Venkatesh B. Cerebral perfusion pressure in neurotrauma: a review. Anesth Analg. (2008) 107:979-88. doi: 10.1213/ane.0b013e31817e7b1a

27. Bordes J, Boret H, Lacroix G, Prunet B, Meaudre E, Kaiser E. Decompressive craniectomy guided by cerebral microdialysis and brain tissue oxygenation in a patient with meningitis. Acta Anaesthesiol Scand. (2011) 55:130-3. doi: 10.1111/j.1399-6576.2010.02355.x

28. van den Brink WA, van Santbrink H, Steyerberg EW, Avezaat CJ, Suazo JA, Hogesteeger C, et al. Brain oxygen tension in severe head injury. Neurosurgery (2000) 46:868-78. doi: 10.1097/00006123-200004000-00018

29. Zweifel C, Lavinio A, Steiner LA, Radolovich D, Smielewski P, Timofeev I, et al. Continuous monitoring of cerebrovascular pressure reactivity in patients with head injury. Neurosurg Focus (2008) 25:E2. doi: 10.3171/FOC.2008.25.10.E2

30. Ho KM, Honeybul S, Yip CB, Silbert BI. Prognostic significance of blood-brain barrier disruption in patients with severe nonpenetrating traumatic brain injury requiring decompressive craniectomy. J Neurosurg. (2014) 121:674-9. doi: 10.3171/2014.6.JNS132838

31. Kamran S, Salam A, Akhtar N, Alboudi A, Kamran K, Singh R, et al. Factors that can help select the timing for decompressive hemicraniectomy for malignant MCA stroke. Transl Stroke Res. (2018) 9:600-7. doi: 10.1007/s12975-018-0616-0

32. Kamran S, Akhtar N, Salam A, Alboudi A, Kamran K, Ahmed A, et al. Revisiting hemicraniectomy: late decompressive hemicraniectomy for malignant middle cerebral artery stroke and the role of infarct growth rate. Stroke Res Treat. (2017) 2017:2507834. doi: 10.1155/2017/2507834

Conflict of Interest Statement: The authors declare that the research was conducted in the absence of any commercial or financial relationships that could be construed as a potential conflict of interest.

Copyright (c) 2019 Shah, Almenawer and Hawryluk. This is an open-access article distributed under the terms of the Creative Commons Attribution License (CC BY). The use, distribution or reproduction in other forums is permitted, provided the original author(s) and the copyright owner(s) are credited and that the original publication in this journal is cited, in accordance with accepted academic practice. No use, distribution or reproduction is permitted which does not comply with these terms. 